\title{
Design of a MIMO Antenna with Improved Isolation Using MNG Metamaterial
}

\author{
Youngki Lee, Deukhyeon Ga, and Jaehoon Choi \\ Department of Electronics and Communications Engineering, Hanyang University, 17 Haengdang-dong, Seongdong-gu, \\ Seoul 133-791, Republic of Korea \\ Correspondence should be addressed to Jaehoon Choi, choijh@hanyang.ac.kr
}

Received 2 April 2012; Accepted 23 May 2012

Academic Editor: Ananda S. Mohan

Copyright ( 92012 Youngki Lee et al. This is an open access article distributed under the Creative Commons Attribution License, which permits unrestricted use, distribution, and reproduction in any medium, provided the original work is properly cited.

A multi-input multi-output (MIMO) antenna with improved isolation using an interdigital split ring resonator (SRR) is proposed. The necessary impedance bandwidth is obtained by utilizing the coupling between the meander strip and an inverted $L$ strip. Using interdigital SRR, a negative permeability was generated, while achieving improved isolation between the two radiating elements. The fabricated antenna satisfies the $10 \mathrm{~dB}$ return loss in the long-term evolution (LTE) band 40 from $2.3 \mathrm{GHz}$ to $2.4 \mathrm{GHz}$. The measured peak gains of the two elements were $2.0 \mathrm{dBi}$ and $1.3 \mathrm{dBi}$. The measured envelope correlation coefficient was less than 0.16 over the frequency band of interest.

\section{Introduction}

Current wireless communication systems require higher bitrate transmission to support various multimedia services. A multi-input multi-output (MIMO) system is regarded as a promising solution, to the problem of data rate transmission since it can increase the channel capacity without sacrificing spectrum efficiency or consuming additional transmitted power [1]. In the MIMO system, two or more antennas are used on both the transmitter and receiver sides. A critical point is to arrange compact antenna elements without impairing antenna performance and system requirements. To do that, mutual coupling or isolation between adjacent antennas is a key factor. However, the antenna elements are strongly coupled with each other as well as with the ground plane, because they share common surface currents. Several techniques have been introduced to improve the isolation characteristic, such as, using the resonating slot on the ground plane [2-6] and using the isolation elements between the radiating elements [7]. Alternative method utilizing the neutralization line was suggested in [8-11]. However, these techniques limit the available space for other system components. Metamaterial (MTM) structures have the ability to concentrate electromagnetic fields and currents near antenna structures instead of spreading them along the antenna ground, which results in higher coupling between antennas. Metamaterial technology has the advantage of reducing the circuit size while providing equivalent or better performance in both antenna and passive circuit applications $[12,13]$. Therefore, the circuit size is independent of the operational frequency and can be significantly reduced to fit in a small area.

This letter proposes a MIMO antenna with improved isolation for the LTE frequency band using the negative permeability property of an interdigital split ring resonator (SRR).

\section{Antenna Design}

2.1. Antenna Structure. The configuration of the proposed MIMO antenna is shown in Figure 1. The proposed MIMO antenna consists of two radiating elements, two inverted $L$ strips, the ground plane, an interdigital SRR, and three FR4 substrates $\left(\varepsilon_{r}=4.4\right)$ with thickness of a $0.4 \mathrm{~mm}$. The two radiating elements of the meander strip shape were printed on the top side of an FR4 substrate (sub \#1), were symmetrically printed with respect to the center, and were placed near the corners of the top edge of the ground plane. The ground plane and two inverted $L$ strips were placed on 


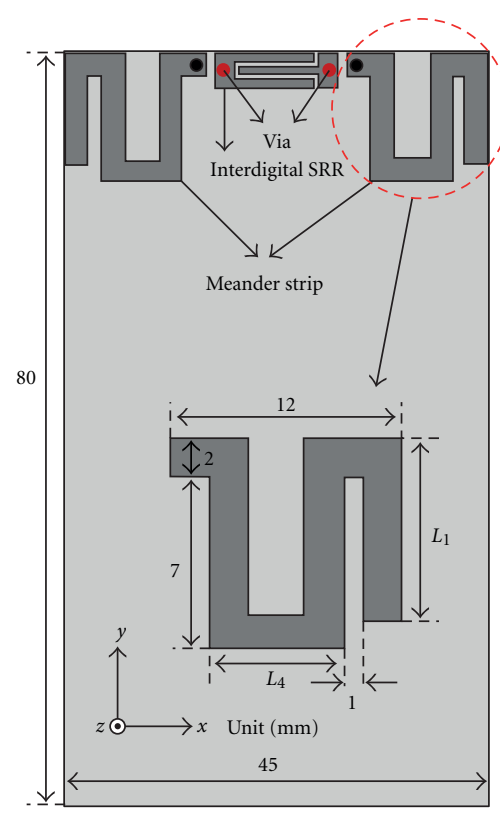

(a)

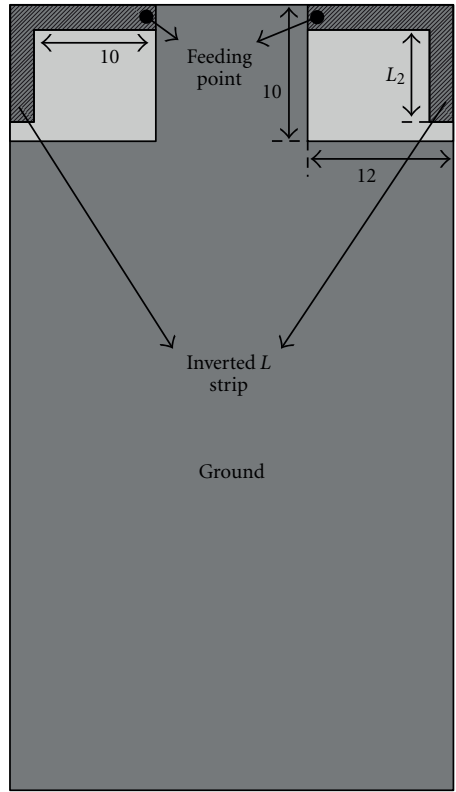

(b)

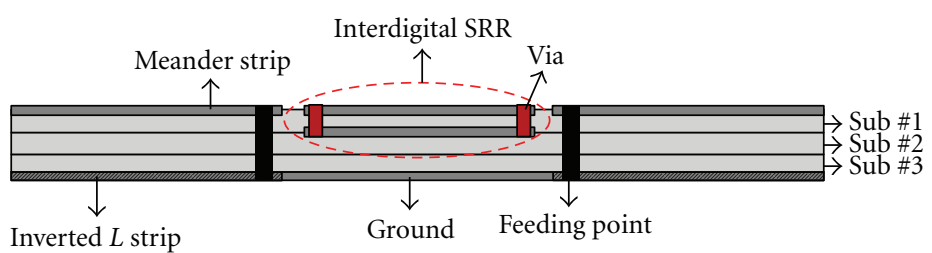

(c)

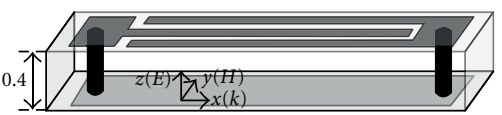

(d)

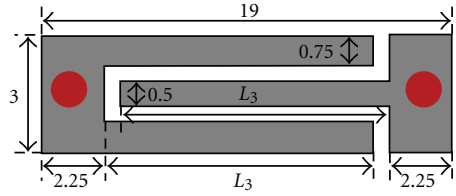

(e)

FIGURE 1: The proposed antenna structure: (a) top view, (b) bottom view, (c) side view, (d) over view of the interdigital SRR, and (e) top view of the interdigital SRR.

the back side of an FR4 substrate (sub \#3). The two inverted $L$ strips were connected to the ground plane. The interdigital SRR was printed on the top and bottom surfaces of the FR4 substrate ( sub \#2) with dimensions of $3 \mathrm{~mm} \times 19 \mathrm{~mm}$ $\times 0.4 \mathrm{~mm}$. The top and bottom surfaces of the interdigital SRR were connected by via holes. The total volume of the proposed MIMO antenna including the two substrates was $45 \mathrm{~mm} \times 80 \mathrm{~mm} \times 1.2 \mathrm{~mm}$.

2.2. Impedance Bandwidth and Isolation. The simulated return loss characteristic with and without the inverted $L$ strip is illustrated in Figure 2(a). To obtain the resonance frequency at the desired band, inverted $L$ strips were printed on the ground plane. The impedance bandwidth can be obtained by utilizing the mutual coupling between the meander strip and the inverted $L$ strip. Figures 2(b) and 2(c) show the simulated return loss characteristics of the proposed antenna. When the length $(L 1)$ of the meander strip and the length $(L 2)$ of the inverted $L$ strip were increased, the resonance frequency shifted toward a lower frequency band. Good impedance matching over LTE band 40 can be achieved by adjusting the sizes of the meander strip and inverted $L$ strip. Figure 2(d) shows the simulated return loss characteristics of the proposed antenna for variation in $L 4$. As $L 4$ increases, the impedance matching near the center frequency region improves. The impedance bandwidth can be easily satisfied by adjusting the length $(L 4)$ of meander strip.

The effective permeability characteristic of the interdigital SRR was extracted using the method suggested in [14]. The negative permeability frequency band of the metamaterial is determined by the capacitance and inductance of the structure.

To analyze the characteristics of the proposed interdigital SRR structure as shown in Figure 1(d), perfect electric 


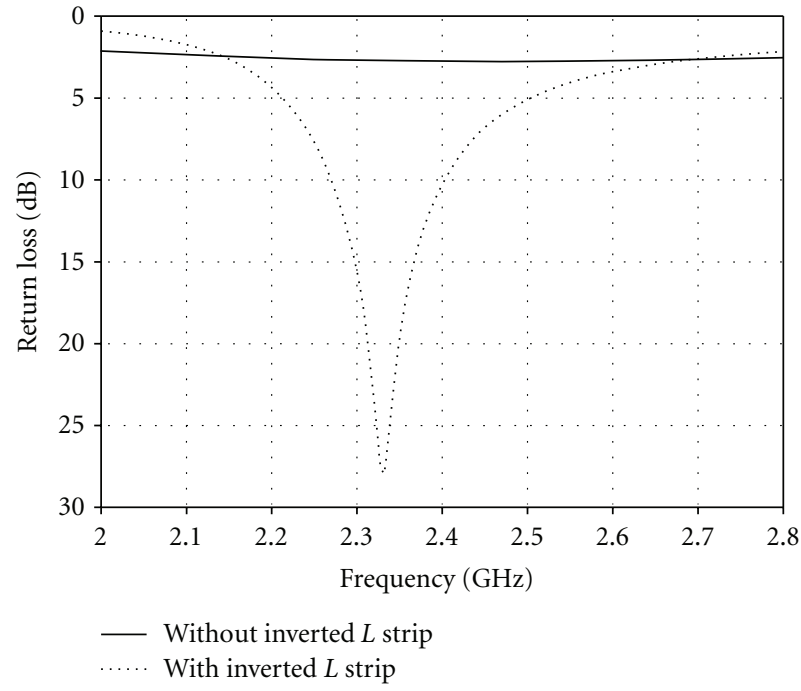

(a)

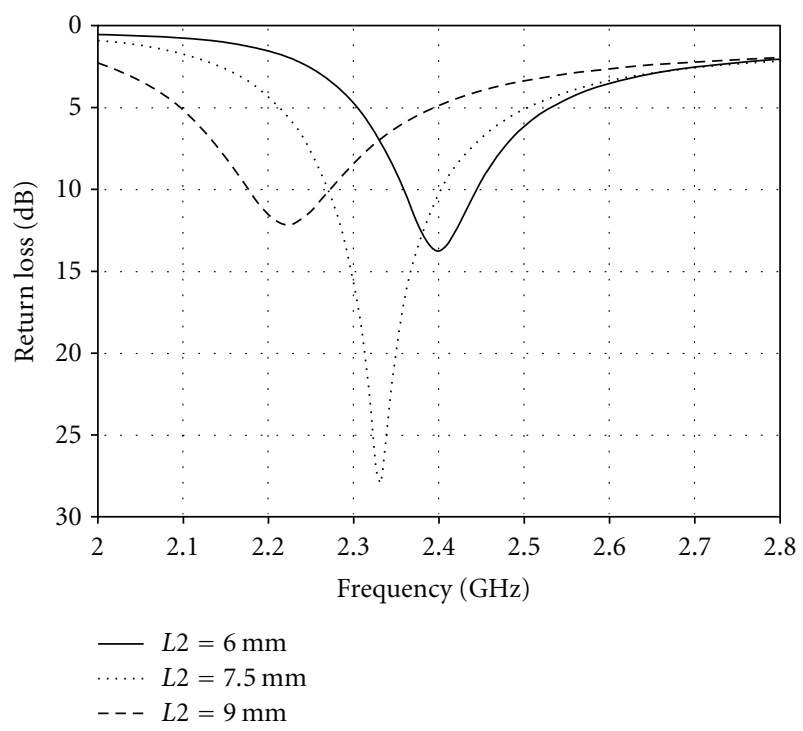

(c)

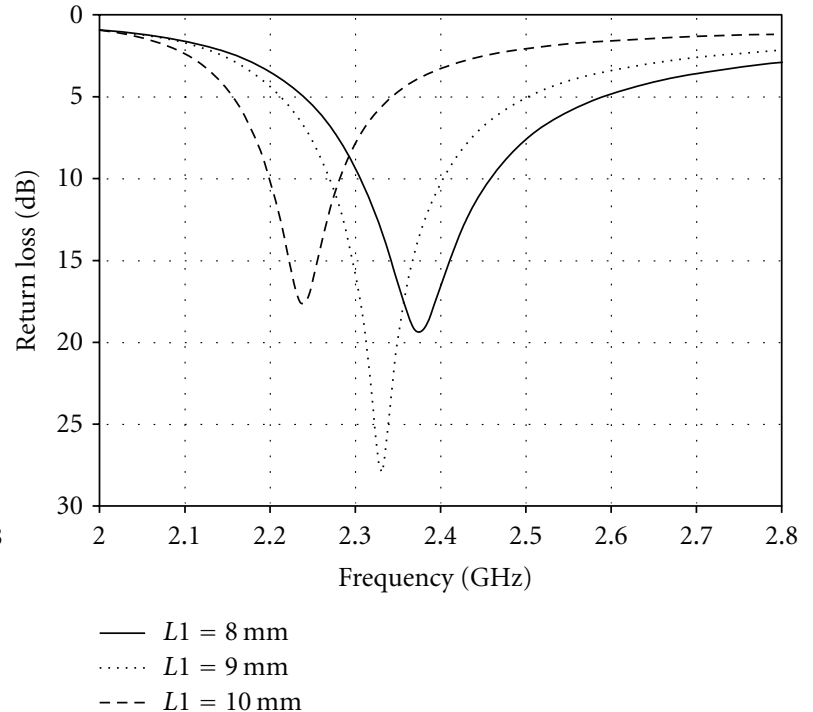

(b)

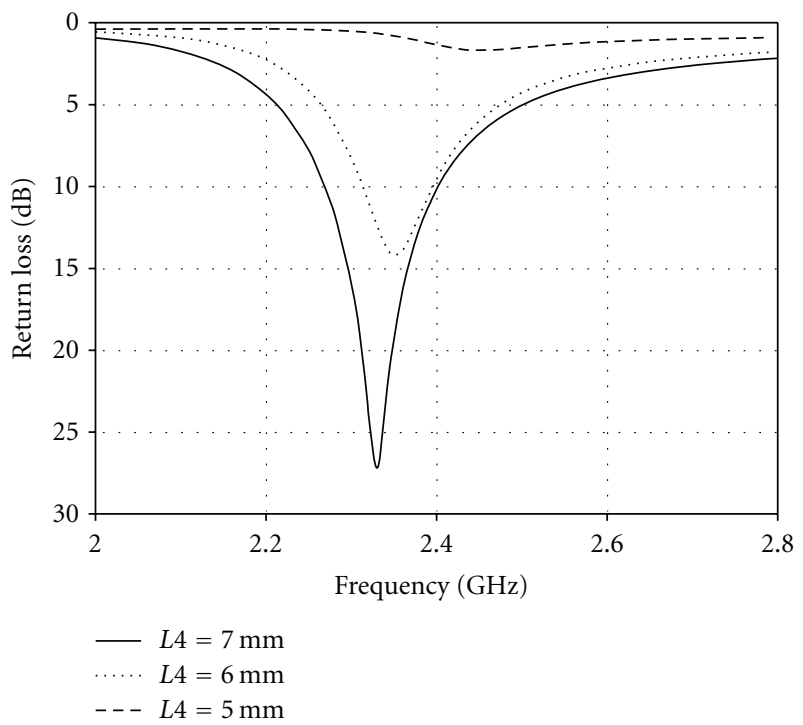

(d)

FIGURE 2: Simulated return loss characteristics: (a) with and without inverted $L$ strip, (b) variation in $L 1$, (c) variation in $L 2$, (d) variation in L4.

conductors (PECs) are placed at the top and bottom (in $x-y$ plane) and perfect magnetic conductors (PMCs) are placed at the front and back (in $x-z$ plane). To obtain the negative magnetic response of the structure, the incident magnetic field is perpendicularly polarized to the $x$ axis and the incident electric field is polarized in parallel with the $z$ axis.

To increase the capacitance, the gap of conventional SRR is replaced by an interdigital structure. By increasing the capacitance, the negative permeability frequency band is shifted from $3.5 \mathrm{GHz}$ to $2.4 \mathrm{GHz}$ without changing the total size of the SRR as shown in Figure 3(a). Figure 3(b) shows the effective permeability characteristics for various lengths of the interdigital SRR (L3). As the length increases, the frequency band of negative permeability shifts to a lower frequency band. Choosing the correct size of the interdigital SRR arm is very important because the resonant frequency is strongly dependent on the length of the interdigital SRR arm.

Figure 4 shows the return loss and isolation characteristics of the proposed MIMO antenna with interdigital SRR for various lengths (L3). When the length of the interdigital SRR is increased, the isolation improvement occurs at the lower frequency without affecting the return loss characteristics over the LTE band 40. From Figures 3(b) and 4(b), one can clearly observe that the negative permeability property of the interdigital SRR improves isolation characteristic of the MIMO antenna systems. 


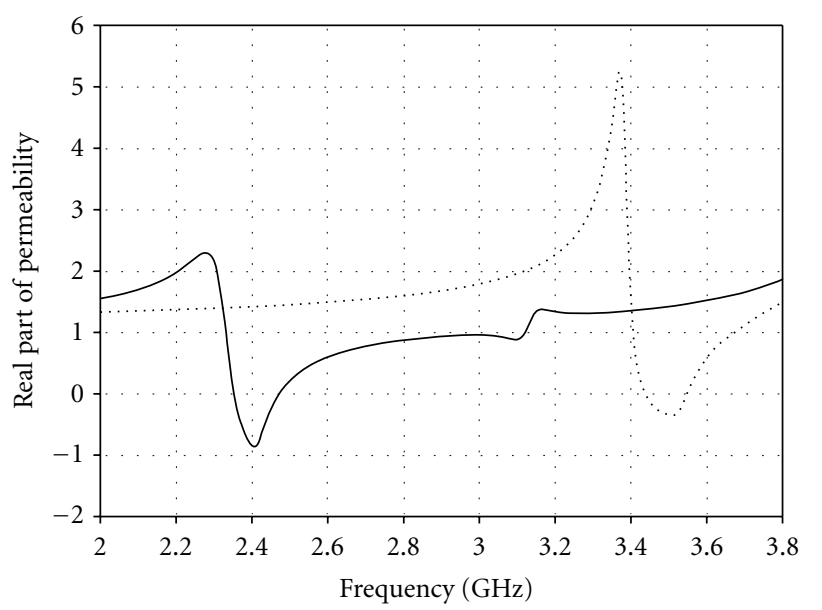

— SRR with interdigital structure … SRR without interdigital structure

(a)

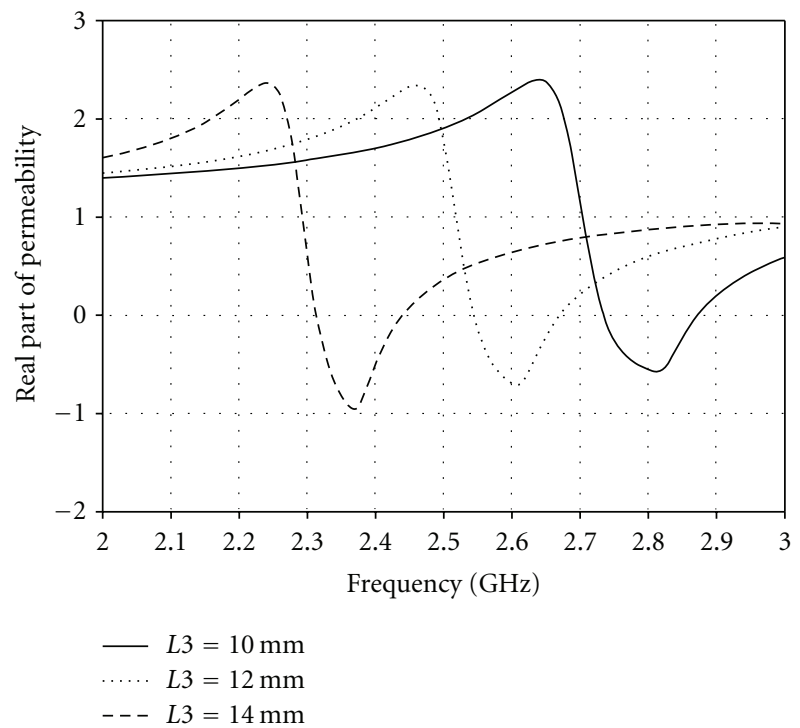

(b)

FIGURE 3: Simulated permeability of the interdigital SRR: (a) comparison between interdigital SRR and conventional SRR, (b) permeability for various $L 3$.

The simulated $S$ parameters with and without the interdigital SRR are illustrated in Figure 5. It is obvious that the addition of the interdigital SRR improves the isolation characteristic. However, due to the narrow negative permeability bandwidth, the impedance matching at the higher frequency band $(2.4 \mathrm{GHz})$ becomes slightly poor. The designed antenna has a $10 \mathrm{~dB}$ return loss bandwidth from $2.27 \mathrm{GHz}$ to $2.41 \mathrm{GHz}$ and an isolation of higher than $20 \mathrm{~dB}$ at the center frequency. It has a negative permeability value at $2.35 \mathrm{GHz}$, so the current could not flow at this frequency. Although the proposed antenna has isolation higher than $15 \mathrm{~dB}$ without the interdigital SRR, the isolation can be improved by approximately $10 \mathrm{~dB}$ at $2.35 \mathrm{GHz}$ by adding the interdigital SRR.

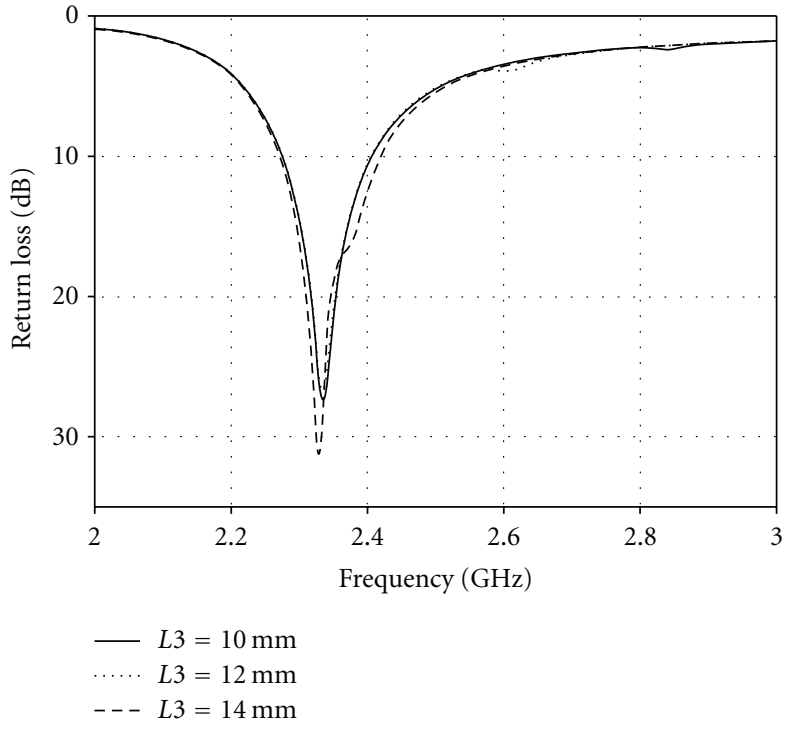

(a)

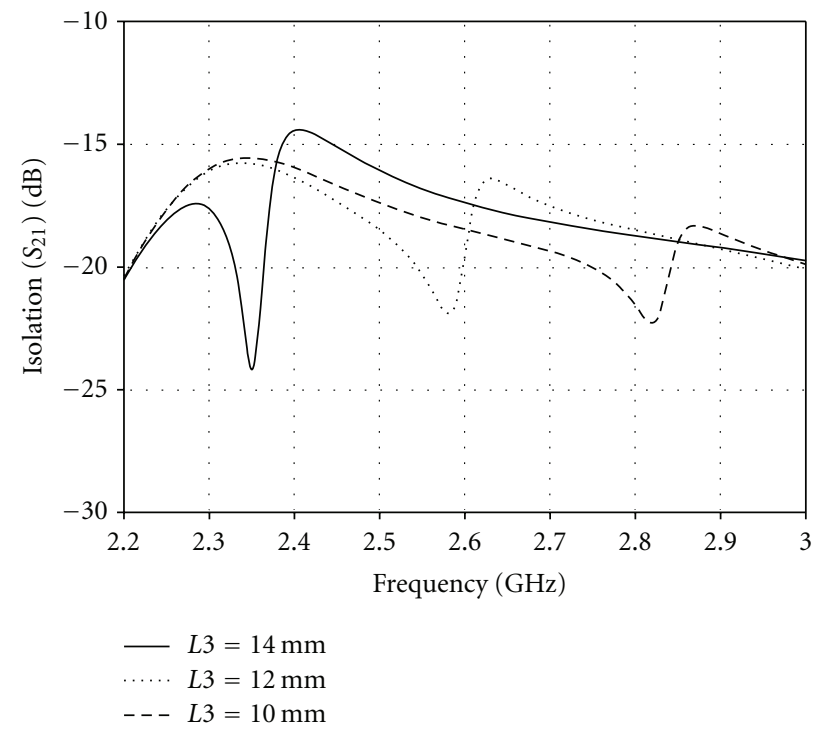

(b)

FIGURE 4: Simulated return loss and isolation characteristics of proposed MIMO antenna with the interdigital SRR: (a) return loss for various $L 3,(\mathrm{~b})$ isolation for various $L 3$.

To investigate the effect of the interdigital SRR on the isolation characteristic, the current distributions at $2.35 \mathrm{GHz}$ with and without the interdigital SRR were calculated and are shown in Figure 6. When one of the two elements was excited, a substantial current was induced at the other element in the absence of an interdigital SRR. After the interdigital SRR was added, the induced current on the nonexcited element became very weak. However, due to the relatively large imaginary part of the permeability of the interdigital SRR which causes losses, the radiation efficiency with interdigital SRR was lower than without it. This fact can also be observed in the current distribution of 


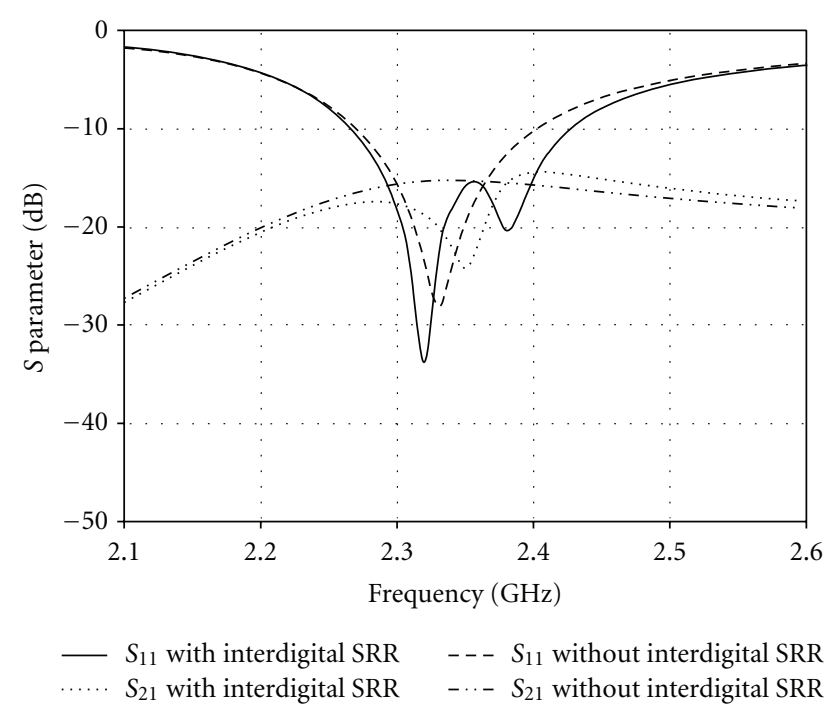

FIGURE 5: Simulated $S$ parameter characteristic of the proposed MIMO antenna.

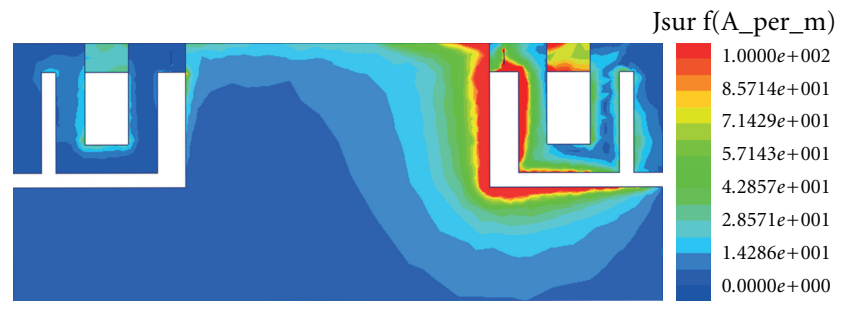

(a) top view

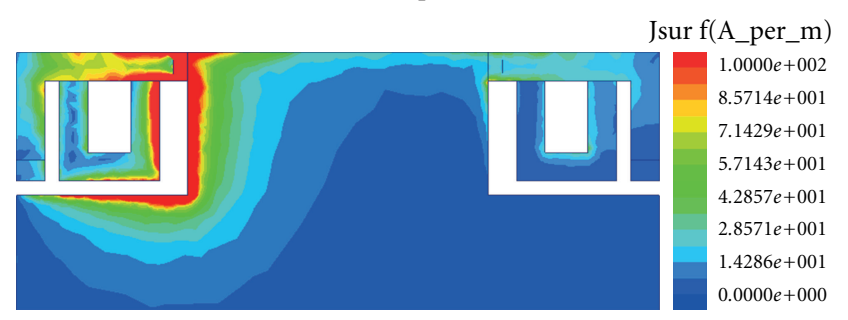

(b) bottom view

Jsur f(A_per_m)

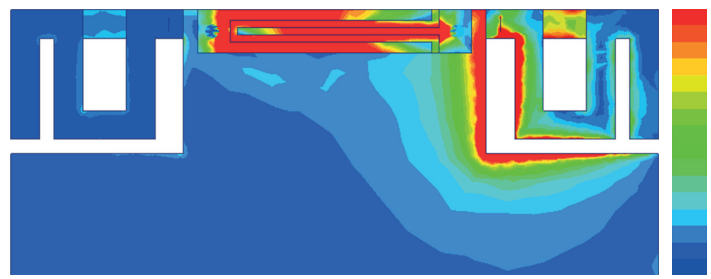

$1.0000 e+002$
$8.5714 e+001$
$7.1429 e+001$
$5.7143 e+001$
$4.2857 e+001$
$2.8571 e+001$
$1.4286 e+001$
$0.0000 e+000$

(c) top view

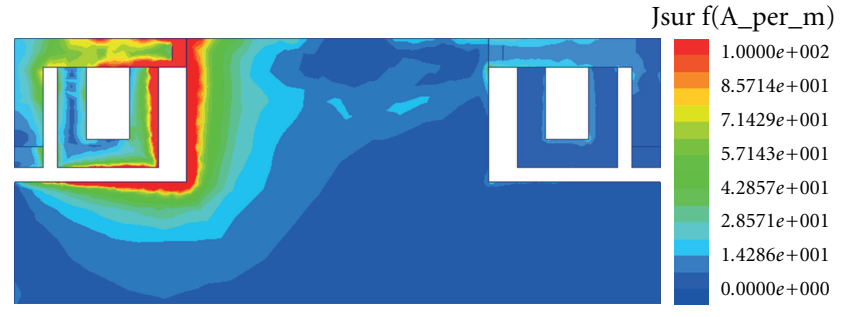

(d) bottom view

FIGURE 6: Simulated current distribution: (a)-(b) without interdigital SRR, (c)-(d) with interdigital SRR.

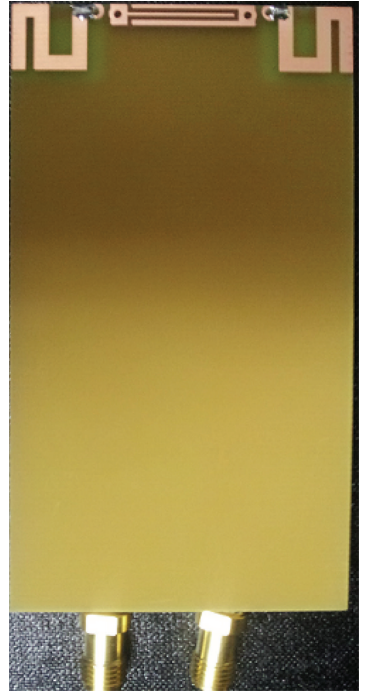

(a)

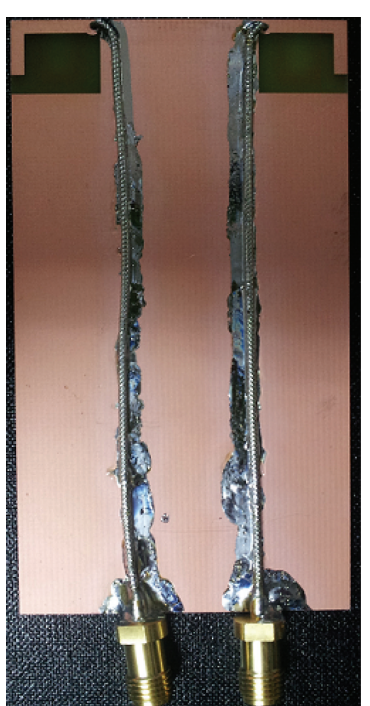

(b)
Figure 7: Photograph of fabricated MIMO antenna: (a) top view, (b) bottom view.

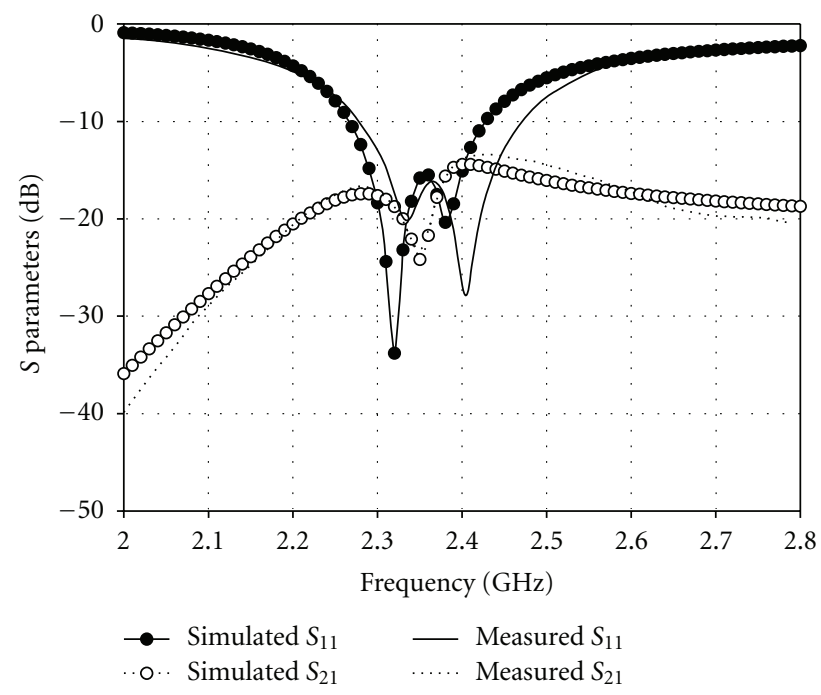

FIGURE 8: Simulated and measured $S$ parameters characteristics.

Figure 6(c). The strong current is induced at the interdigital SRR. The antenna structure was designed and analyzed using a high-frequency structure simulator (HFSS V13.) [15].

\section{Measurements}

A photograph of the fabricated antenna is shown in Figure 7. The measured and simulated return loss values in terms of frequency are compared in Figure 8. The measured results were very similar to the simulated results. The fabricated antenna has a $10 \mathrm{~dB}$ return loss bandwidth over the whole LTE band $40(2.3 \sim 2.4 \mathrm{GHz})$ and an isolation higher than $20 \mathrm{~dB}$ at the center frequency.

The measured radiation patterns of the two radiating elements of the designed MIMO antenna are shown in 

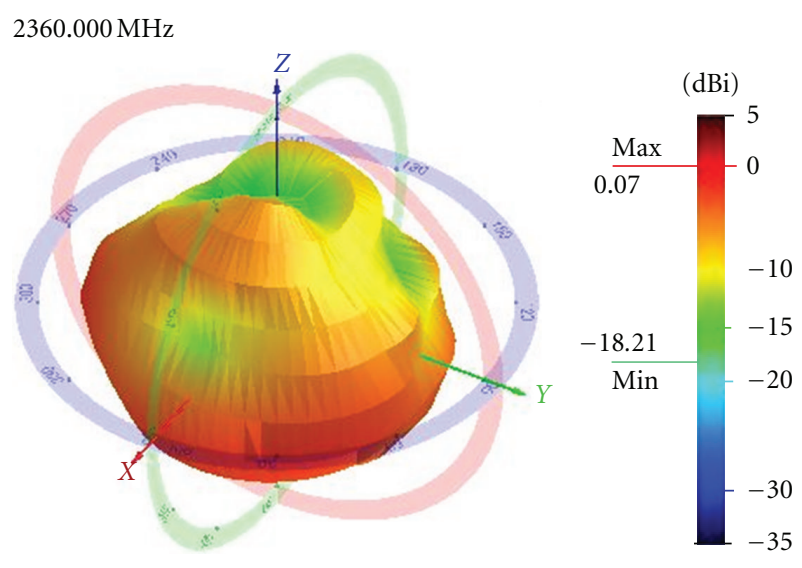

(a)
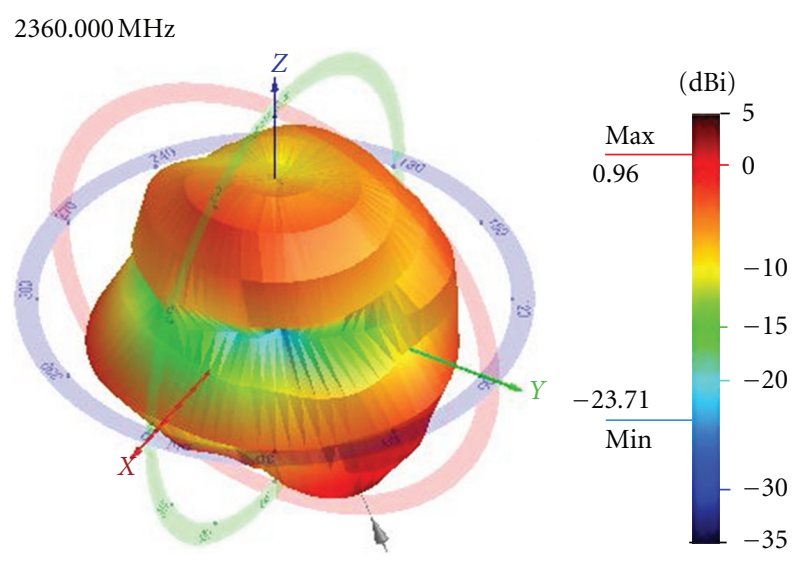

(b)

FIGURE 9: Measured radiation pattern: (a) radiator \#1, (b) radiator $\# 2$.

Figure 9. The radiation patterns are symmetrical with respect to the $X$-axis and each element has near omni-directional radiation patterns at the operating frequency. Figure 10 shows the peak gains and radiation efficiencies of the two elements at the operating frequency. The peak gains were $2.0 \mathrm{dBi}$ and $1.3 \mathrm{dBi}$, and the measured average radiation efficiencies were $34 \%$ and $37 \%$, respectively.

For diversity and MIMO applications, the correlation between signals received at the same side of a wireless link by the involved antenna is an important figure of merit for the whole system. Usually, the envelope correlation coefficient (ECC) is used to evaluate the diversity capability of a multiantenna system. This parameter should preferably be computed from $3 \mathrm{D}$ radiation patterns, but this method is laborious. Assuming that an antenna operates in a uniform multipath environment, this parameter can alternatively be computed from its scattering parameters. The ECC of the two antennas is given by [16]:

$$
\mathrm{ECC}=\frac{\left|S_{11}^{*} S_{12}+S_{12}^{*} S_{12}\right|^{2}}{\left(1-\left|S_{11}\right|^{2}-\left|S_{21}\right|^{2}\right)\left(1-\left|S_{22}\right|^{2}-\left|S_{12}\right|^{2}\right)} .
$$

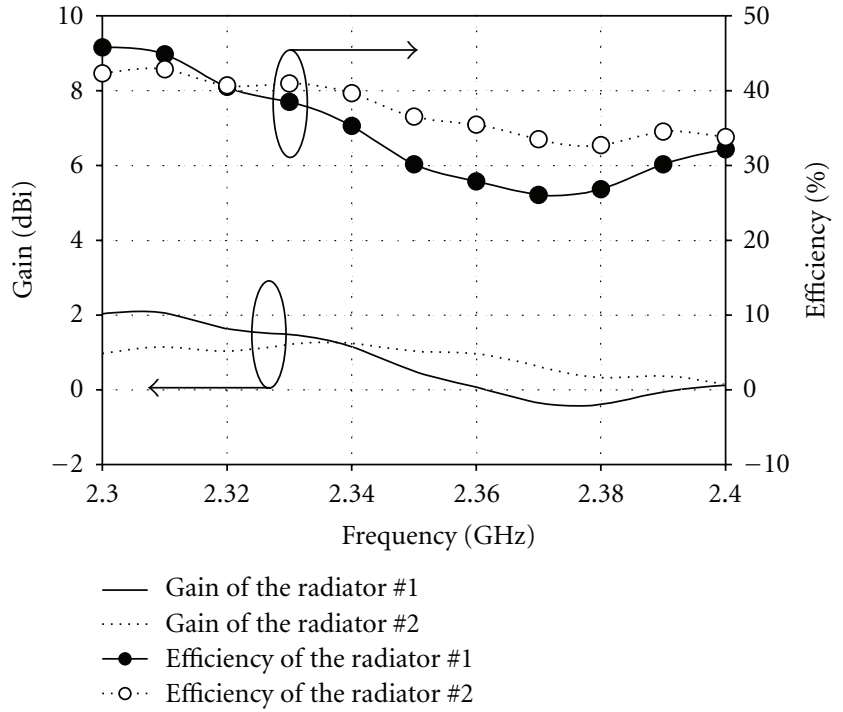

Figure 10: Measured gain and radiation efficiency characteristics.

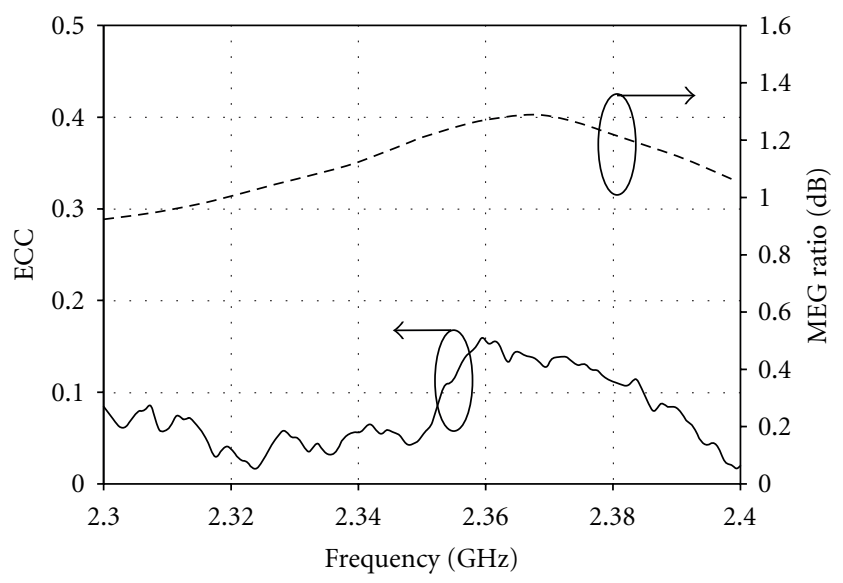

FIGURE 11: Measured ECC and MEG ratio characteristics.

Figure 11 shows the ECC and mean effective gain (MEG) ratio characteristics computed from the measured scattering parameters. The ECC of the two antennas was less than 0.16, and the measured MEG ratio was less than $1.12 \mathrm{~dB}$ over the whole LTE band 40 .

\section{Conclusion}

In this letter, we proposed a MIMO antenna with improved isolation for the LTE frequency band using the negative permeability property of an interdigital SRR. The impedance bandwidth was achieved by utilizing electromagnetic coupling between the meander strip and the inverted $L$ strip.

The interdigital SRR structure was added between the two elements to improve the isolation characteristic. The return loss was higher than $10 \mathrm{~dB}$ and the isolation characteristic was higher than $15 \mathrm{~dB}$ over the whole LTE band 40 . The measured peak gains of the two elements were $2.0 \mathrm{dBi}$ 
and $1.3 \mathrm{dBi}$. The measured envelope correlation coefficient was less than 0.16 and the mean effective gain ratio was less than $1.2 \mathrm{~dB}$. Therefore, the interdigital SRR structure can be used in MIMO antenna systems to obtain the high-isolation characteristic necessary for mobile applications.

\section{Acknowledgment}

This work was supported by the national research foundation of Korea (NRF) Grant funded by the Korea government (MEST) (no. 2011-0016495).

\section{References}

[1] D. Gesbert, M. Shafi, D. S. Shiu, P. J. Smith, and A. Naguib, "From theory to practice: an overview of MIMO space-time coded wireless systems," IEEE Journal on Selected Areas in Communications, vol. 21, no. 3, pp. 281-302, 2003.

[2] C. Y. Chiu, C. H. Cheng, R. D. Murch, and C. R. Rowell, "Reduction of mutual coupling between closely-packed antenna elements," IEEE Transactions on Antennas and Propagation, vol. 55, no. 6, pp. 1732-1738, 2007.

[3] K. J. Kim, W. G. Lim, and J. W. Yu, "High isolation internal dual-band planar inverted-F antenna diversity system with band-notched slots for MIMO terminals," in Proceedings of the 36th European Microwave Conference (EuMC '06), pp. 14141417, September 2006.

[4] W.-S. Chen, C.-H. Lin, B.-Y. Lee, W.-H. Hsu, and F.-S. Chang, "Monopole slot antenna design for WLAN MIMO application," Microwave and Optical Technology Letters, vol. 54, no. 4, pp. 1103-1107, 2012.

[5] J. Rahola and J. Ollikainen, "Removing the effect of antenna matching in isolation analyses using the concept of electromagnetic isolation," in Proceedings of the IEEE International Workshop on Antenna Technology: Small Antennas and Novel Metamaterials (IWAT '08), pp. 554-557, March 2008.

[6] J.-F. Li, Q.-X. Chu, and T.-G. Huang, "A compact wideband MIMO antenna with two novel bent slits," IEEE Transactions on Antennas and Propagation, vol. 60, no. 2, pp. 482-489, 2012.

[7] C. H. Lee, S. Y. Chen, and P. Hsu, "Integrated dual planar inverted-F antenna with enhanced isolation," IEEE Antennas and Wireless Propagation Letters, vol. 8, pp. 963-965, 2009.

[8] B. Y. Park, J. H. Choi, S. O. Park, T. S. Yang, and J. H. Byun, "Design of a decoupled MIMO antenna for LTE applications," Microwave and Optical Technology Letters, vol. 53, no. 3, pp. 582-586, 2011.

[9] Y. Liu, W. Jiang, S. Gong, and L. Jiang, "Novel miniaturized and high-isolation MIMO antenna," Microwave And Optical Technology Letters, vol. 54, no. 2, pp. 511-515, 2012.

[10] J. Rahola and J. Ollikainen, "Analysis of isolation of two-port antenna systems using simultaneous matching," in Proceedings of the 2nd European Conference on Antennas and Propagation (EuCAP '07), Edinburgh, UK, November 2007.

[11] S.-W. Su, C.-T. Lee, and F.-S. Chang, "Printed MIMO-antenna system using neutralization-line technique for wireless USBdongle applications," IEEE Transactions on Antennas and Propagation, vol. 60, no. 2, pp. 456-463, 2012.

[12] C. Caloz and T. Itoh, Electromagnetic Matamaterial: Transmission Line Theory and Microwave Applications, the Engineering Approach, John Wiley \& Sons, New York, NY, USA, 2005.

[13] C. J. Lee, K. M. K. H. Leong, and T. Itoh, "Composite right/left-handed transmission line based compact resonant antennas for RF module integration," IEEE Transactions on Antennas and Propagation, vol. 54, no. 8, pp. 2283-2291, 2006.

[14] D. R. Smith, D. C. Vier, T. Koschny, and C. M. Soukoulis, "Electromagnetic parameter retrieval from inhomogeneous metamaterials," Physical Review E, vol. 71, no. 3, Article ID 036617, pp. 1-11, 2005.

[15] Ansoft High Frequency Structure Simulator (HFSS), Ver.12, ANSYS Inc.

[16] S. Blanch, J. Romeu, and I. Corbella, "Exact representation of antenna system diversity performance from input parameter description," Electronics Letters, vol. 39, no. 9, pp. 705-707, 2003. 

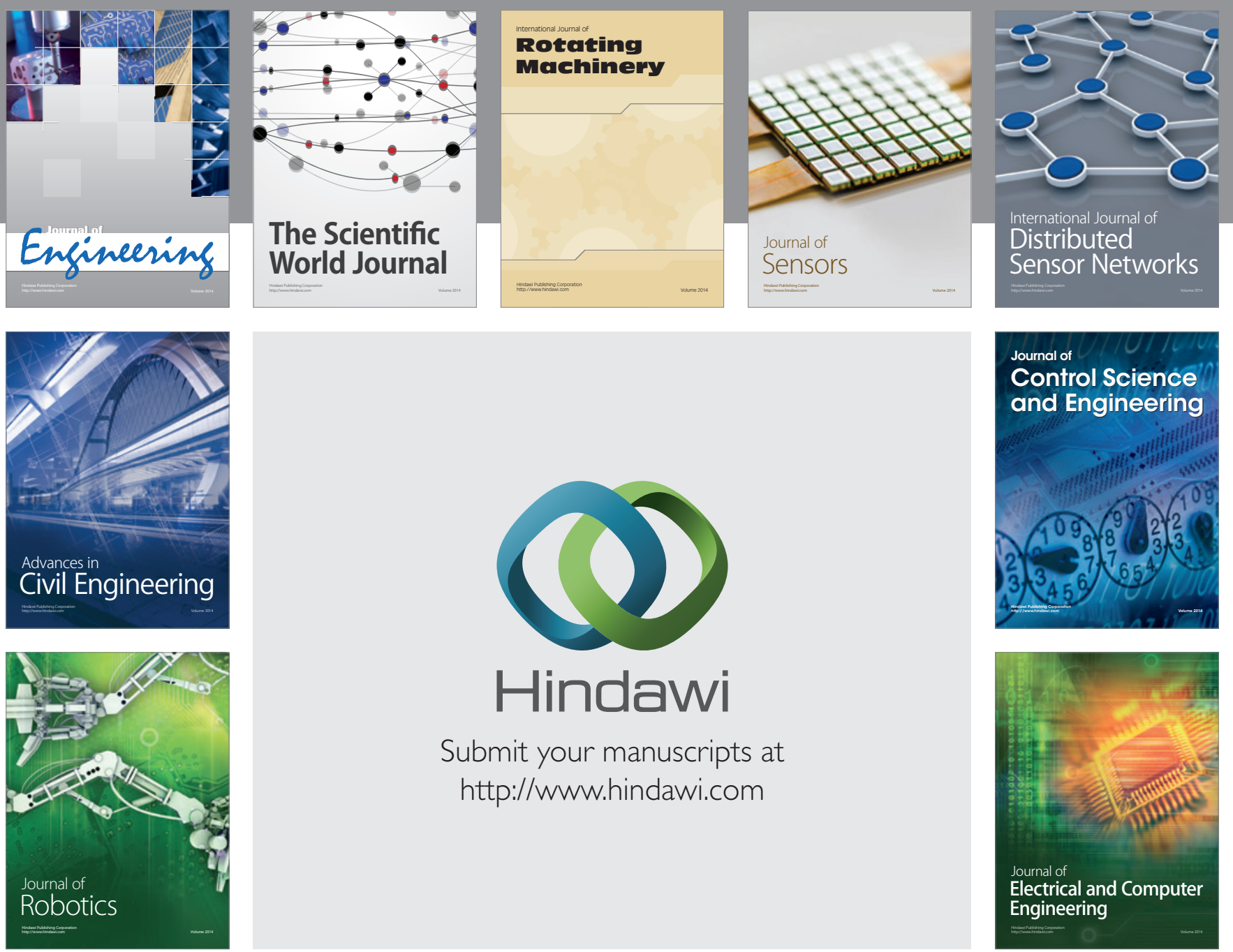

Submit your manuscripts at

http://www.hindawi.com
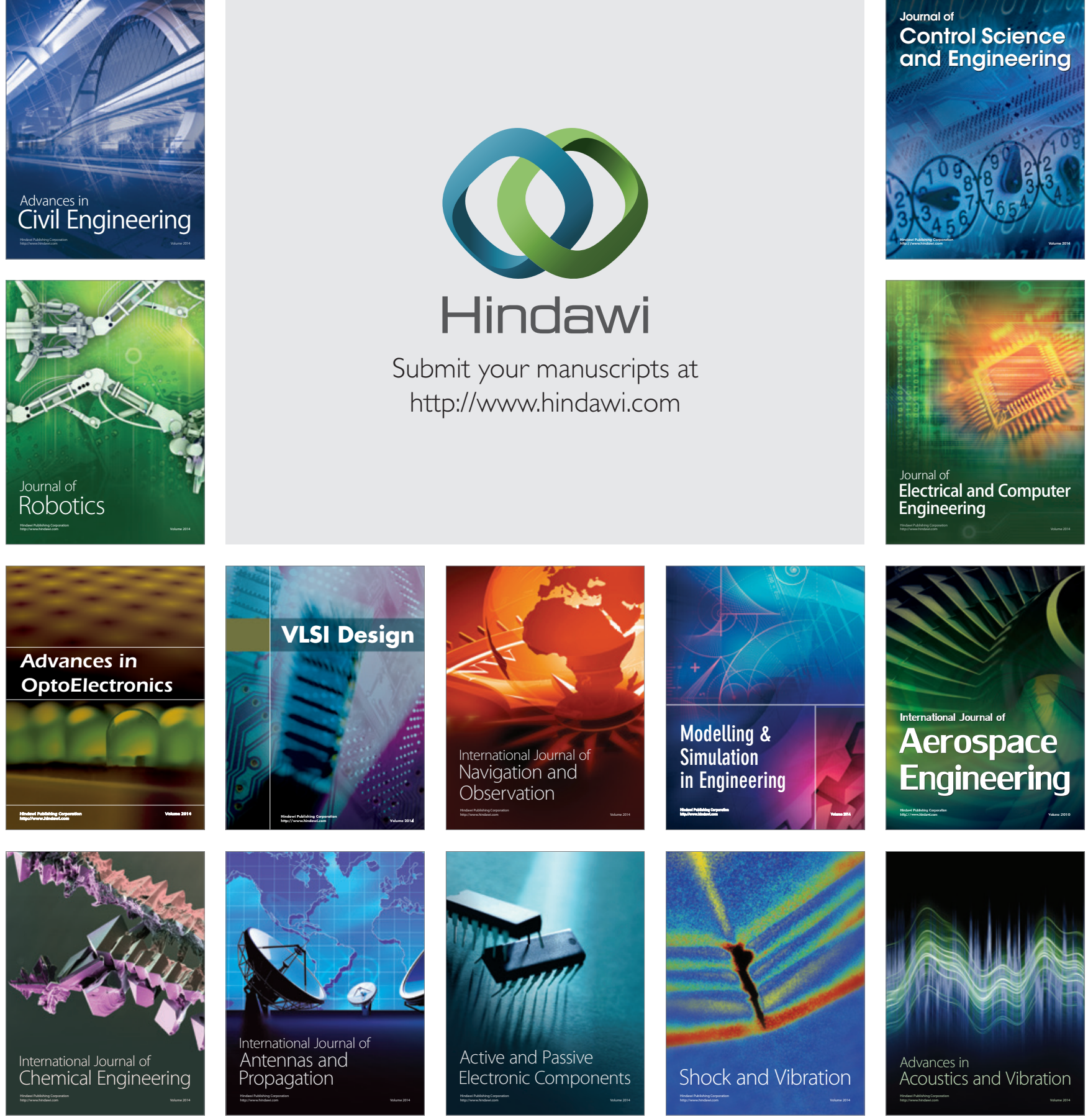